\title{
Robustness of SOC Estimation Algorithms for EV Lithium-Ion Batteries against Modeling Errors and Measurement Noise
}

\author{
Xue Li, ${ }^{1,2}$ Jiuchun Jiang, ${ }^{1,2}$ Caiping Zhang, ${ }^{1,2}$ Le Yi Wang, ${ }^{3}$ and Linfeng Zheng ${ }^{1,2}$ \\ ${ }^{1}$ National Active Distribution Network Technology Research Center (NANTEC), Beijing Jiaotong University, Beijing 100044, China \\ ${ }^{2}$ Collaborative Innovation Center of Electric Vehicles in Beijing, Beijing Jiaotong University, Beijing 100044, China \\ ${ }^{3}$ Department of Electrical and Computer Engineering, Wayne State University, Detroit, MI 48202, USA \\ Correspondence should be addressed to Jiuchun Jiang; jcjiang@bjtu.edu.cn
}

Received 16 March 2015; Revised 1 August 2015; Accepted 9 August 2015

Academic Editor: Dongsuk Kum

Copyright (C) 2015 Xue Li et al. This is an open access article distributed under the Creative Commons Attribution License, which permits unrestricted use, distribution, and reproduction in any medium, provided the original work is properly cited.

\begin{abstract}
State of charge (SOC) is one of the most important parameters in battery management system (BMS). There are numerous algorithms for SOC estimation, mostly of model-based observer/filter types such as Kalman filters, closed-loop observers, and robust observers. Modeling errors and measurement noises have critical impact on accuracy of SOC estimation in these algorithms. This paper is a comparative study of robustness of SOC estimation algorithms against modeling errors and measurement noises. By using a typical battery platform for vehicle applications with sensor noise and battery aging characterization, three popular and representative SOC estimation methods (extended Kalman filter, PI-controlled observer, and $H_{\infty}$ observer) are compared on such robustness. The simulation and experimental results demonstrate that deterioration of SOC estimation accuracy under modeling errors resulted from aging and larger measurement noise, which is quantitatively characterized. The findings of this paper provide useful information on the following aspects: (1) how SOC estimation accuracy depends on modeling reliability and voltage measurement accuracy; (2) pros and cons of typical SOC estimators in their robustness and reliability; (3) guidelines for requirements on battery system identification and sensor selections.
\end{abstract}

\section{Introduction}

Electric vehicles (EVs), including hybrid electric vehicles (HEVs), battery electric vehicles (BEVs), and plug-in hybrid electric vehicles (PHEVs), have become a critical driving force for green economy and attracted great research effort recently. An appropriate battery management system (BMS) is indispensable for safe, reliable, and efficient operations of EV battery systems [1]. The state of charge (SOC) is one of the most important state variables in BMS. Failure to estimate SOC accurately may cause overdischarging or overcharging, resulting in decreased battery longevity and even causing dangerous accidents [2].

There are many methods to estimate the SOC, with their own pros and cons. The Coulomb integral method [3] is easy to implement, but it needs the prior knowledge of the initial SOC and suffers from accumulated errors from measurement noise and bias. The open circuit voltage (OCV) method is a more reliable approach for SOC estimation. There is a monotonic relationship between the OCV and SOC. However, this relationship is accurate only at a steady-state after several hours of open circuit condition. As a result, the OCV method cannot be used reliably for online SOC estimation [4]. The Kalman filter [5, 6] and extended Kalman filter (EKF) [7-12] have the appealing property of minimizing the mean-square estimation errors when the state and output measurement noises are additive, independent, zero mean, and Gaussian. On the other hand, they are susceptible to modeling errors and noise feature variations. The nonlinear observer method [13] employs a feedback mechanism to correct SOC estimation errors. Although this method works well under noise-free environment, its feedback gain must be carefully designed to achieve noise attenuation and robustness, which are highly challenging.

The accuracy of the model parameters is one of the main reasons affecting the SOC estimation accuracy. In [14], the researchers analyze the effects of the model parameters on the SOC estimation accuracy, when the model parameters drift 
due to battery aging. Various degrees of impact of different model parameters are established, leading to some parameter updating guidelines to focus on high-impact parameters so that computational complexity can be reduced.

The paper is a comparative study of several typical SOC estimation algorithms on their robustness against modeling errors and measurement noises. We focus on variations of model parameters caused by battery aging. Our simulation results demonstrate that the Ohmic resistance $R_{\mathrm{O}}$, polarization resistance $R_{P}$, and the open circuit voltage $\mathrm{OCV}$ are the key parameters affecting SOC estimation accuracy. However, the polarization capacitor $C_{P}$ which is an important parameter only influences the dynamic response characteristics of SOC estimation and does not have noticeable effects on the steady-state accuracy of SOC estimation. Within the extended Kalman filter algorithm, $H_{\infty}$ observer, and PI observer studied in this paper, our results indicate that the robustness and the estimation accuracy of the three methods against modeling errors and measurement noises are similar. However, in the view of application and SOC accuracy, the PI observer has advantages over the $H_{\infty}$ observer and the EKF algorithm to be applied in BMS.

This paper reveals that SOC estimation accuracy depends critically on voltage measurement errors. While random noises in voltage measurements can be effectively filtered out, any bias or persistent errors will cause substantial deterioration on SOC estimation accuracy, which is a major reason for many algorithms to fail. Since voltage measurement accuracy varies substantially among BMS manufacturers, careful examination and enhancement of robustness of algorithms by design improvement and online parameter estimation are of essential importance. This paper utilizes some common scenarios of battery aging and parameter variations to study this issue and provide some related guidelines on how to select a robust method which has a strong tolerance towards voltage measurement errors.

The main contributions of this paper are in the following aspects which are essential for BMS design: (1) a clear analysis of the influence of each model parameter on the SOC estimation precision; (2) comparison of the robustness of various SOC estimation algorithms against model errors; (3) establishment of the quantitative relationship between measurement noise and SOC estimation accuracy.

The remainder of this paper is organized as follows. The battery model is introduced in Section 2. The three observerbased algorithms under study are described. Estimation accuracy of these algorithms is evaluated in Section 3 and their robustness is quantitatively compared. Section 4 investigates adaptability of the three algorithms against system uncertainties. Finally, some conclusions are drawn in Section 5.

\section{Battery Model}

Lithium-ion battery is a complex, nonlinear electrochemical system. It is difficult to find a very accurate model to describe the complex changes in its charging and discharging processes. Extensive research on batteries has generated many battery models [15-19]. In these battery models, the equivalent circuit model is used commonly, including the

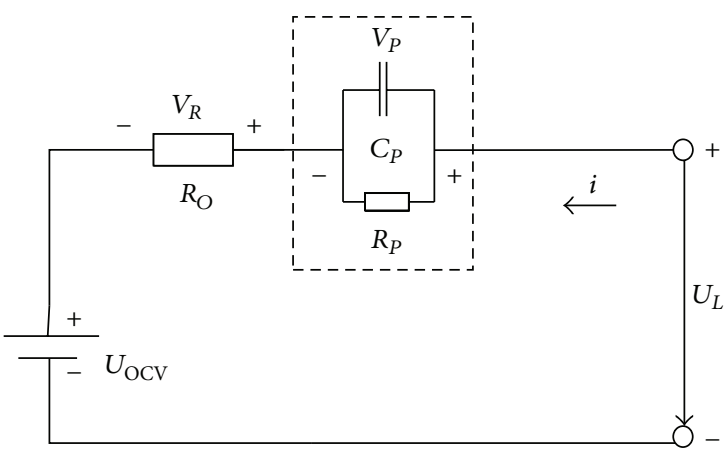

FiguRE 1: First-order RC model.

Rint model, the first-order RC model, and the second-order RC model $[9,20]$. In general, an accurate battery model is essential for precise battery state estimation. However, high-fidelity battery models need more complex structures, more parameters, and carry high computational complexity. Therefore, it is necessary to find a compromise between accuracy of SOC estimation and simplicity of the model. In this paper, a first-order RC model is employed, shown in Figure 1, in which the battery terminal voltage error is within $\pm 20 \mathrm{mV}$, meeting the requirements of the estimation accuracy.

One of the most important state variables in BMS is state of charge (SOC), which is defined as

$$
\operatorname{SOC}(t)=\operatorname{SOC}_{0}+\frac{1}{Q} \int_{0}^{t} \eta I(\tau) d \tau,
$$

where $\operatorname{SOC}(t)$ is the SOC at time $t, \mathrm{SOC}_{0}$ the initial value, $Q$ the battery nominal capacity, $I(\tau)$ the current at time $\tau$, and $\eta$ the coulomb efficiency. The coulomb efficiency can be considered to be 1 [21,22]. The influence of the self-discharge on battery SOC estimation can be neglected.

According to Kirchhoff's current and voltage laws, it is easy to obtain the following mathematical relationships:

$$
\begin{aligned}
& U_{L}=U_{\mathrm{OCV}}+I R_{\mathrm{O}}+V_{P}, \\
& \dot{V}_{P}=-\frac{1}{R_{P} C_{P}} V_{P}+\frac{1}{C_{P}} I,
\end{aligned}
$$

where $R_{O}$ is the Ohmic resistance, $R_{P}$ the polarization resistance, $C_{P}$ the polarization capacitor, $U_{\mathrm{OCV}}$ the open circuit voltage, $U_{L}$ the terminal voltage, $V_{R}$ the voltage across $R_{O}$, and $V_{P}$ the polarization voltage.

According to (1), it can be converted into the derivative equation as follows:

$$
\mathrm{SOCC}=\frac{I}{Q} .
$$

The relationship between the SOC and OCV is nonlinear. In this paper, this function is represented by piecewise linear segments,

$$
U_{\mathrm{OCV}_{i}}=k_{i} \mathrm{SOC}_{i}+b_{i}=f(\mathrm{SOC}),
$$

where $k_{i}$ is the slope of the $i$ th line segment and $b_{i}$ is the intercept. Their values are listed as in Table 1. 
TABLE 1: The values of $k_{i}$ and $b_{i}$.

\begin{tabular}{lcccccccccc}
\hline$i$ & 1 & 2 & 3 & 4 & 5 & 6 & 7 & 8 & 9 \\
\hline SOC $_{i}$ & $0-7$ & $7-12$ & $12-17$ & $17-22$ & $22-27$ & $27-31$ & $31-36$ & $36-41$ & $41-46$ & $46-51$ \\
$k_{i}$ & 6.48 & 1.75 & 0.60 & 0.64 & 0.52 & 0.49 & 0.60 & 0.59 & 0.44 & 0.41 \\
$b_{i}$ & 3.20 & 3.53 & 3.67 & 3.66 & 3.69 & 3.69 & 3.66 & 3.67 & 3.73 & 3.74 \\
\hline$i$ & 11 & 12 & 13 & 14 & 15 & 16 & 17 & 18 & 19 & 20 \\
\hline SOC $_{i}$ & $51-56$ & $56-61$ & $61-66$ & $66-71$ & $71-76$ & $76-80$ & $80-85$ & $85-90$ & $90-95$ & $95-100$ \\
$k_{i}$ & 0.40 & 0.32 & 0.29 & 0.28 & 0.39 & 0.50 & 0.37 & 0.38 & 0.52 & 1.05 \\
$b_{i}$ & 3.75 & 3.79 & 3.81 & 3.82 & 3.74 & 3.66 & 3.76 & 3.75 & 3.62 & 3.12 \\
\hline
\end{tabular}

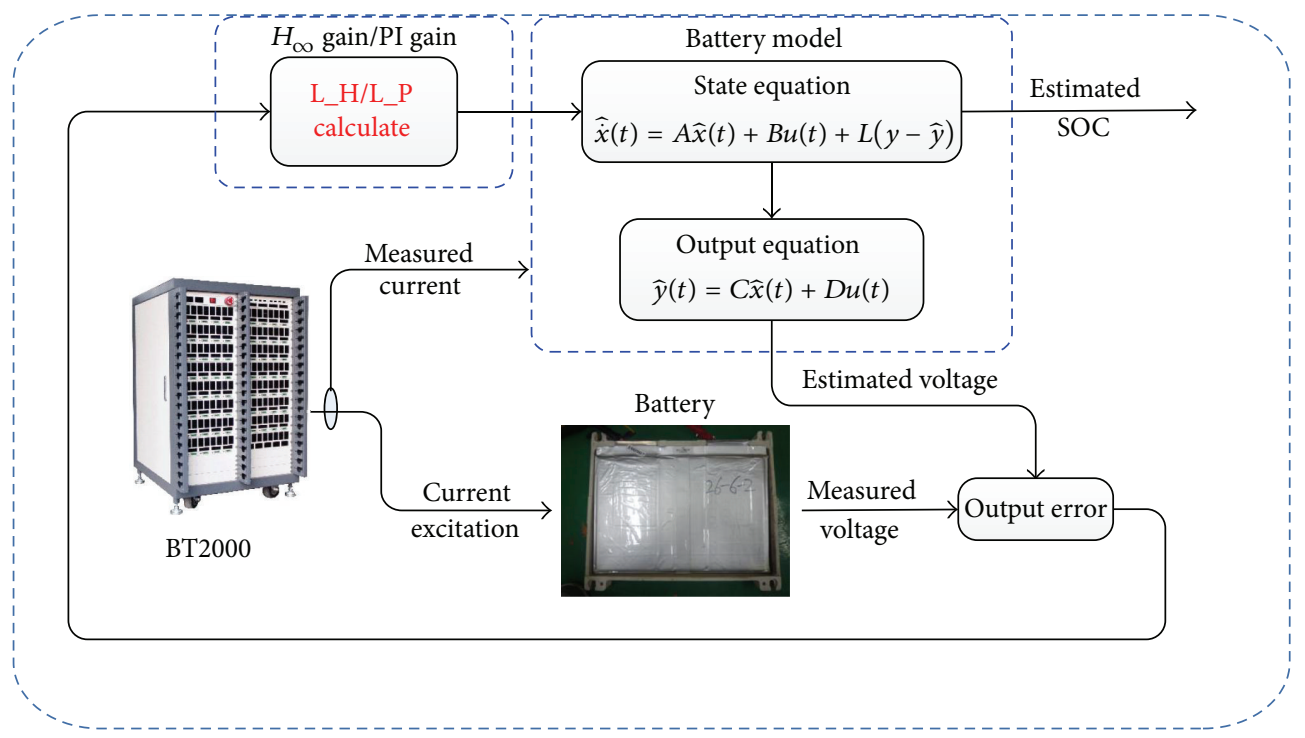

FIGURE 2: The general structure of the closed-loop observers.

If the state of the battery is defined as $x=\left[V_{P}, \mathrm{SOC}\right]^{T}$, then the state equations of the battery in each segment are linear

$$
\begin{aligned}
& \dot{x}(t)=A x(t)+B u(t), \\
& y(t)=C x(t)+D u(t),
\end{aligned}
$$

where $A=\left[\begin{array}{cc}-1 / R_{P} C_{P} & 0 \\ 0 & 0\end{array}\right], B=\left[\begin{array}{ll}1 / C_{P} & 1 / Q\end{array}\right]^{T}, C=\left[\begin{array}{ll}1 & k_{i}\end{array}\right], D=$ $R_{O}, u(t)=I(t)$, and $y(t)=U_{L}(t)$.

\section{SOC Estimation Algorithms and Their Robustness}

In this paper, three closed-loop observers are evaluated on their accuracy and robustness in SOC estimation. The key control principle of the closed-loop observers is to use the difference between the measured terminal voltage and the estimated value as the input to the feedback module with a gain matrix to update the polarization voltage $V_{P}$ and SOC. The general structure of the closed-loop observers is shown in Figure 2.

3.1. The $H_{\infty}$ Observer. The $H_{\infty}$ control theory was initiated by Zames in his seminal paper [23]. Since then extensive theoretical development, efficient solutions using frequency domain methods, state space models, numerical algorithms, and software packages have resulted in a rich treatise in this field [24-26]. Numerous successful applications have also been documented. In particular, numerical solutions to standard $H_{\infty}$ observers can be found by using the Robust Control Tool Box and LMI model in MATLAB.

The main advantages of the $H_{\infty}$ observer are as follows: (1) it is designed to attenuate disturbances of broader types than Kalman filters and Wiener filters which target Gaussian white noises; (2) it is robust against unstructured model uncertainty. However, as a worst-case robust design approach, it may be conservative, namely, nonoptimal, if the noise spectrum is actually known.

Consider a generic nonlinear battery system described by

$$
\begin{aligned}
& \dot{x}(t)=A x(t)+B u(t)+F \lambda, \\
& y(t)=C x(t)+D u(t)+E(t)+G \lambda,
\end{aligned}
$$

where $A, B, C, D, E, F$, and $G$ are coefficient matrices, which depend on the actual battery system, $x(t)$ is the state, and $y(t)$ is the output. Consider $E(t)=b_{i}(t), \lambda=\left[\begin{array}{ll}\omega & \nu\end{array}\right]^{T}, \omega=\left[\begin{array}{c}\omega_{1} \\ \omega_{2}\end{array}\right]$, $F=\left[\begin{array}{lll}1 & 0 & 0 \\ 0 & 1 & 0\end{array}\right]$, and $G=\left[\begin{array}{lll}0 & 0 & 1\end{array}\right]$. 
The structure of the observer is

$$
\begin{aligned}
& \hat{\dot{x}}(t)=A \hat{x}(t)+B u(t)+L(y-\hat{y}), \\
& \hat{y}(t)=C \hat{x}(t)+D u(t)+E(t),
\end{aligned}
$$

where $\widehat{x}(t)$ and $\widehat{y}(t)$ are the estimates for $x(t)$ and $y(t)$ and $L$ is the gain vector of the observer.

Define the state estimation error $e(t)=x(t)-\widehat{x}(t)$. Then the error dynamics is

$$
\dot{e}(t)=\dot{x}(t)-\hat{\dot{x}}(t)=(A-L C) e(t)+(F-L G) .
$$

The goal of the observer design is disturbance attenuation: for a given (acceptable) sensitivity coefficient $\gamma>0$, design the observer gain $L$ such that the error system (10) is stable and that the following inequality is met under the zero initial condition:

$$
\|e(t)\| \leq \gamma\|\lambda(t)\| .
$$

The gain $L=P^{-1} X$ may be numerically solved by using the LMI approach [27]

$$
\begin{gathered}
\min \left(\gamma^{2}\right) \\
P>0 \\
{\left[\begin{array}{cc}
A^{T} P-C^{T} X^{T}+P A-X C+I & P F-X G \\
(P F-X G)^{T} & -\gamma^{2}
\end{array}\right]<0,}
\end{gathered}
$$

where $P=P^{T}, X=P L$, for which the LMI Toolbox in MATLAB can be used. The derivation of (12) is similar to the derivation in [13].

Reference [13] has verified that the battery state space model (8) is observable. As a result, the LMI approach is applicable to design the $H_{\infty}$ observer to estimate the SOC of the battery system.

3.2. The PI Observer. The proportional control law is the simplest most common control law. However, it carries steady-state error which limits its applications alone. The integral controller is not only related to the size of the input bias but also related to the existence of time deviation. As long as the bias exists, the output will continue to accumulate until the bias is zero; it will stop accumulating. Therefore, the integral control can eliminate steady-state error. Although the integral control can eliminate residual error, it slows down the control action, and as such it has detrimental effect on stability and transient performance. By combining these two control actions, the proportional and integral (PI) control inherits the advantages of both.

The observability and stability of the PI observer estimating the battery SOC are proved in [28]. Therefore, the PI observer can be utilized to estimate the battery SOC.

The relationship of the input and the output in the PI observer is

$$
\begin{aligned}
\varphi(t) & =K_{p}\left[e(t)+\frac{1}{T_{i}} \int e(t) d t\right] \\
& =K_{p} e(t)+K_{i} \int e(t) d t,
\end{aligned}
$$

where $K_{p}$ is the proportional gain of the observer, $T_{i}$ the time constant of integration, $K_{i}$ the integral gain of the observer, $\varphi(t)$ the output of the feedback system in the observer, and $e(t)$ the error between the estimated voltage and measured value, which is the input to the feedback loop in the observer.

The Ziegler-Nichols tuning method [29] is a heuristic method of tuning a PID controller. It was developed by Nichols and Ziegler. It is performed by setting the integral gain $K_{i}$ to zero. The proportional gain $K_{p}$ is then increased (from zero) until it reaches the ultimate gain $K_{u}$, at which the output of the control loop oscillates with a constant amplitude. The ultimate gain $K_{u}$ and the oscillation period $T_{u}$ are used to set the proportional gain $K_{p}$ and the integral gain $K_{i}$. According to [29], the proportional gain $K_{p}$ and the integral gain $K_{i}$ in the simulation are set as follows:

$$
\begin{gathered}
K_{p}=1.5, \\
K_{i}=0.3 .
\end{gathered}
$$

The PI observer takes the advantage of the proportional control to generate control action immediately and that of the integral control to eliminate residual error. The control parameters must be properly designed to achieve a desirable balance between dynamic quality and steady-state performance of the observer.

3.3. The Extended Kalman Filter. In 1960, Kalman published his famous paper describing a recursive solution to optimal discrete-time linear filtering problems under additive and independent Gaussian noise [30]. A Kalman filter estimates the state of a dynamic system with a linear process model and measurement model [31]. Its extension to nonlinear systems employs local linearization, leading to the extended Kalman filter (EKF) [32].

In its application to the battery systems considered in this paper, the discrete-time nonlinear system with additive noise is given by

$$
\begin{aligned}
& {\left[\begin{array}{c}
V_{P}(k+1) \\
\operatorname{SOC}(k+1)
\end{array}\right]} \\
& =\left[\begin{array}{cc}
\exp \left(-\frac{\Delta t}{R_{P} C_{P}}\right) & 0 \\
0 & 1
\end{array}\right]\left[\begin{array}{c}
V_{P}(k) \\
\operatorname{SOC}(k)
\end{array}\right] \\
& +\left[\begin{array}{c}
R_{P}\left(1-\exp \left(-\frac{\Delta t}{R_{P} C_{P}}\right)\right) \\
\frac{\Delta t}{Q}
\end{array}\right] I(k)+\left[\begin{array}{c}
\omega_{1} \\
\omega_{2}
\end{array}\right] .
\end{aligned}
$$

By substituting (5) into (2), the terminal voltage can be expressed as

$$
U_{L}(k)=k_{i} \operatorname{SOC}(k)+I(k) R_{O}+V_{P}(k)+b_{i}+v(k) .
$$


Therefore, coefficient matrices can be derived as

$$
\begin{aligned}
A & =\left[\begin{array}{cc}
\exp \left(-\frac{\Delta t}{R_{P} C_{P}}\right) & 0 \\
0 & 0
\end{array}\right] \\
B & =\left[\begin{array}{c}
R_{P}\left(1-\exp \left(-\frac{\Delta t}{R_{P} C_{P}}\right)\right) \\
\frac{\Delta t}{Q}
\end{array}\right], \\
C & =\left[\begin{array}{ll}
1 & k_{i}
\end{array}\right], \\
D & =R_{O} .
\end{aligned}
$$

The EKF algorithm involves the following steps:

(i) Prediction update:

$$
\begin{aligned}
& \widehat{x}_{k}^{-}=A \widehat{x}_{k-1}+B I_{k-1}, \\
& P_{k}^{-}=A P_{k-1} A^{T}+\varepsilon .
\end{aligned}
$$

(ii) Measurement update:

$$
\begin{aligned}
\widehat{x}_{k} & =\widehat{x}_{k}^{-}+K_{k}\left(U_{L}(k)-\widehat{U}_{L}(k)\right), \\
K_{k} & =P_{k}^{-} C^{T}\left[C P_{k}^{-} C^{T}+\delta\right]^{-1}, \\
P_{k} & =\left[1-K_{k} C\right] P_{k}^{-},
\end{aligned}
$$

where $\varepsilon$ and $\delta$ are the variances of the noises $\omega(k)$ and $\nu(k)$, respectively, and $\widehat{x}_{k}^{-}$is the updated state estimate from the previous estimate $\widehat{x}_{k-1}$.

Implementation of the EKF is depicted by the flowchart in Figure 3.

3.4. Experiments. In this paper, one battery with a nominal capacity of $92 \mathrm{Ah}$, whose anode is lithium manganese oxide and whose cathode is graphite, is used in our experiments to verify parameter identification and SOC estimation accuracy. All experiments are accomplished on the battery testing platform, shown in Figure 4, which includes the Arbin Testing System, thermostat, PC, BMS, and a high precision multimeter. The charge and discharge tests are finished by the Arbin Instrument BT2000 battery testing system, whose maximum voltage and charge/discharge current are $5 \mathrm{~V}$ and $400 \mathrm{~A}$, respectively, in which the current can be set to the low range $(-1 \mathrm{~A} \sim 1 \mathrm{~A})$, the middle range $(-50 \mathrm{~A} \sim 50 \mathrm{~A})$, or the high range ( $-400 \mathrm{~A} \sim 400 \mathrm{~A}$ ), according to the required maximum testing current. The controllable temperature range of the thermostat is $-373.15 \mathrm{~K} \sim 233.15 \mathrm{~K}$. The BMS is manufactured by Huizhou Epower Electronic Co., Ltd. The digital multimeter with a 6.5-digit resolution has a precision of $0.1 \mathrm{mV}$, so the voltage measured by this device is considered as true values. The noise is acquired by subtracting the BMS measured value from the digital multimeter measured value, whose statistical distribution is shown in Figure 5. The statistical characteristics of the terminal voltage measurement noise of the BMS are specified in Table 2.
TABLE 2: Statistical properties of terminal voltage and current measurement noise.

\begin{tabular}{lcc}
\hline & Mean & Variance \\
\hline $\begin{array}{l}\text { The terminal } \\
\text { voltage }\end{array}$ & $1.3 \times 10^{-3} \mathrm{~V}$ & $4.1368 \times 10^{-7} \mathrm{~V}^{2}$ \\
$\begin{array}{l}\text { measurement noise } \\
\text { of the BMS }\end{array}$ & \\
\hline
\end{tabular}

The tests are composed of two parts. One part is parameter identification experiment and the other part is SOC estimation accuracy verification experiment. A 1/3 C constant current is used to charge the battery to $4.2 \mathrm{~V}$, and the capacity $C$ in this paper refers to the maximum available capacity of the battery in current state of health if not figured out specifically. These experiments were first performed when the battery was brand-new. When the capacity of the battery was reduced to $74.5 \mathrm{Ah}$, the experiments were repeated to analyze the impact of the model parameter variations caused by battery aging. Data points are acquired at $1 \mathrm{~Hz}$ during the tests.

The battery model is built on the MATLAB/Simulink platform. The measured data including the voltage and current from the test bench are used as the input information to the three algorithms to estimate the SOC value. The SOC estimation accuracies are compared with the current integration values which serve as the SOC reference values.

The current integration method has two disadvantages: (a) it needs to know the initial SOC value in advance; (b) there is an accumulated error caused by the current measurement that is not accurate.

First, in this paper, the battery is discharged entirely in advance, so the initial SOC value is 0 . Therefore, the initial SOC value is known. Second, the data used in the paper is obtained by the Arbin Instrument BT2000 battery testing system, and the precision of the current is $0.1 \%$ of the measuring range. The current range in experiments is set to the middle range $(-50 \mathrm{~A} \sim 50 \mathrm{~A})$, so the precision of the current sampling is $0.1 \mathrm{~A}$. When the battery is charged in $1 / 3 \mathrm{C}$ current rate, the maximum SOC accumulated error in one full charging is $(0.1 \mathrm{~A} * 3 \mathrm{~h}) / 92 \mathrm{Ah} * 100 \%=0.32 \%$, which can be neglected. Therefore, the SOC value calculated by the current integration method in instrument can serve as the SOC reference values in this paper, which overcomes the shortcomings of the current integration method effectively.

3.5. Verification. The SOC is estimated from experimental data by using the three estimation methods, respectively. Figure 6(a) shows the results of SOC estimation from the $H_{\infty}$ observer; Figure 6(b) shows the results of SOC estimation from the PI observer; and Figure 6(c) shows the results of SOC estimation from the EKF algorithm. The left $y$-axis is the SOC value, including the estimated value and experimental data, and the right $y$-axis is the SOC estimation error, and the $x$-axis is the time. All of three observers demonstrate good SOC estimation accuracy and convergence to the true SOC value, with a very short response time. 


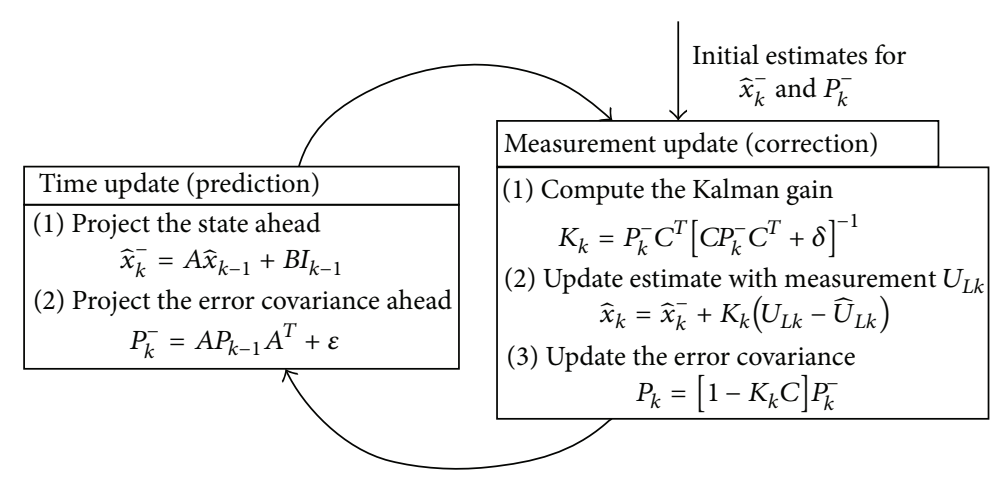

FIgURE 3: The operation of EKF.

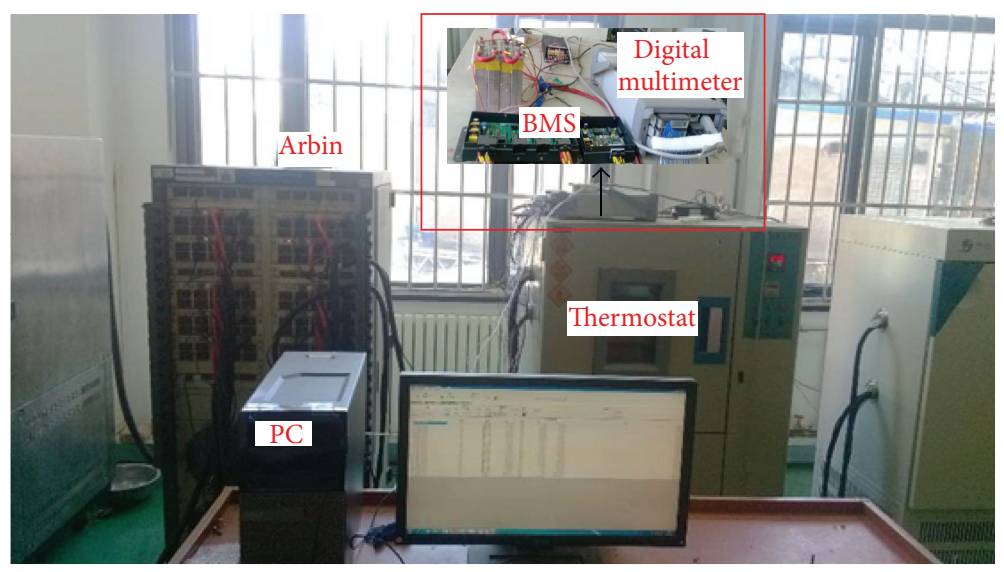

FIGURE 4: The battery testing platform.

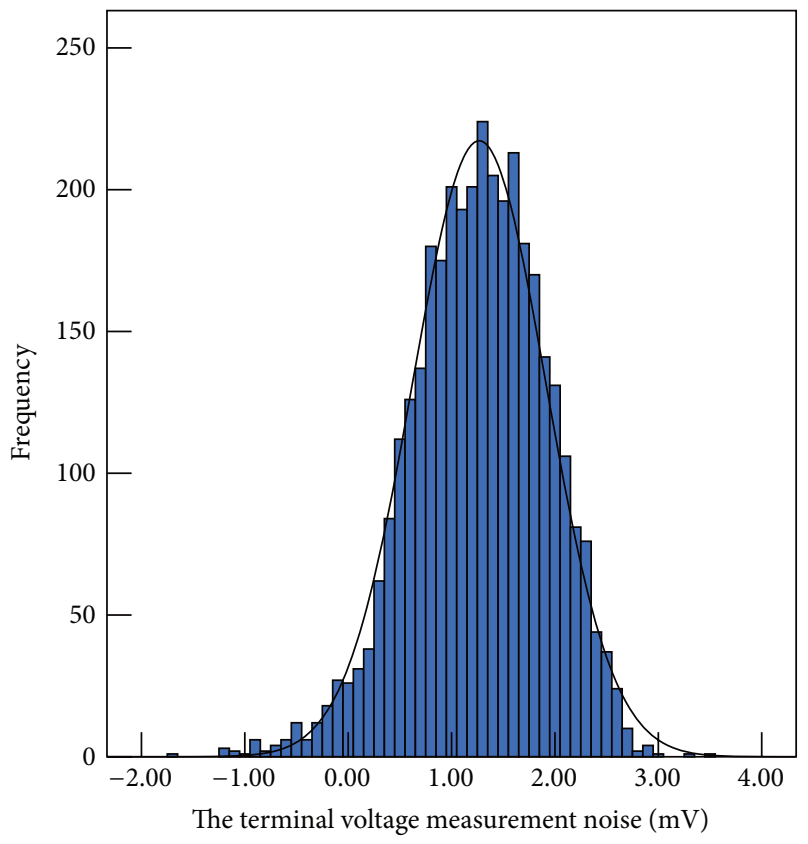

FIGURE 5: The statistical properties of the terminal voltage measurement.
While the SOC estimation errors of the three estimation methods have the same trends, there are important differences among them, as indicated by Figure 6(d). In Figure 6(d), it is apparent that the EKF algorithm has the largest transient volatility, which is partially due to the initial value of $P_{k}^{-}, \varepsilon$, and $\delta$. After SOC estimation reaches steadystate, the $H_{\infty}$ observer has the largest SOC estimation errors, with the upper limit $1.67 \%$ and lower limit $-0.79 \%$ implying that the $H_{\infty}$ observer is least accurate. This may be attributed to the fact that the $H_{\infty}$ observer is a conservative estimation method, which does not attenuate noise optimally.

The steady-state SOC estimation errors of the three estimation methods can maintain between the $2 \%$ band and $-2 \%$ band when the SOC initial error is $20 \%$, see Figure $6(d)$.

\section{Robust Analysis of the Algorithms against System Uncertainties}

In this section, the adaptability of the noise characteristics is discussed in detail. The noise is divided into two parts. One is the modelling error due to parameter changes caused by battery aging and the other is the terminal voltage measurement noise caused by BMS sampling accuracy. The 


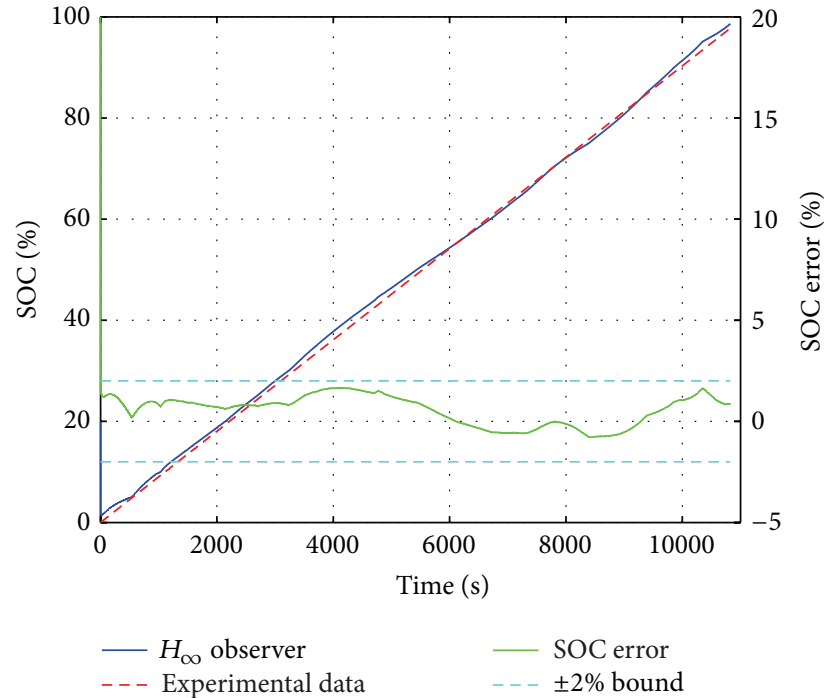

(a) $H_{\infty}$ observer

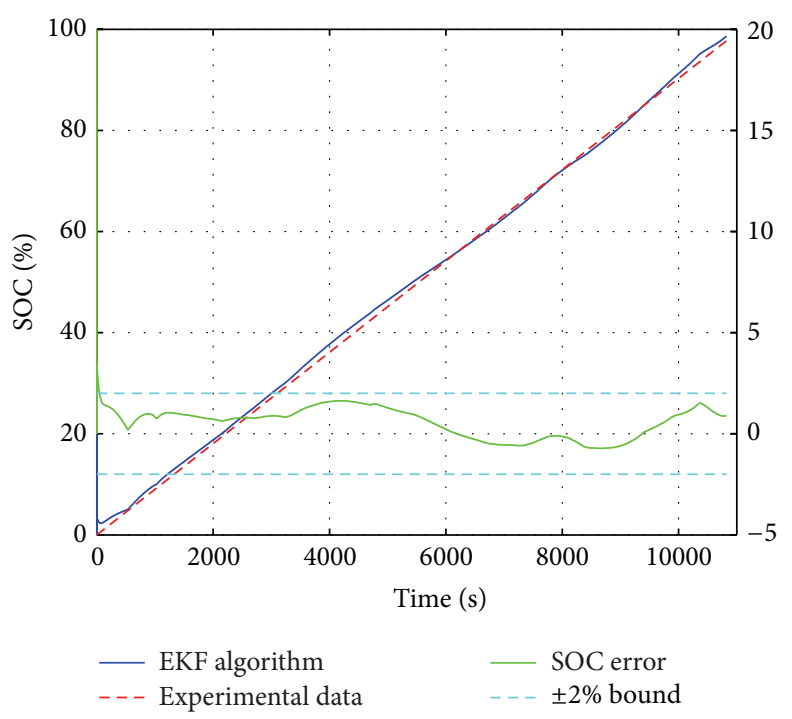

(c) EKF algorithm

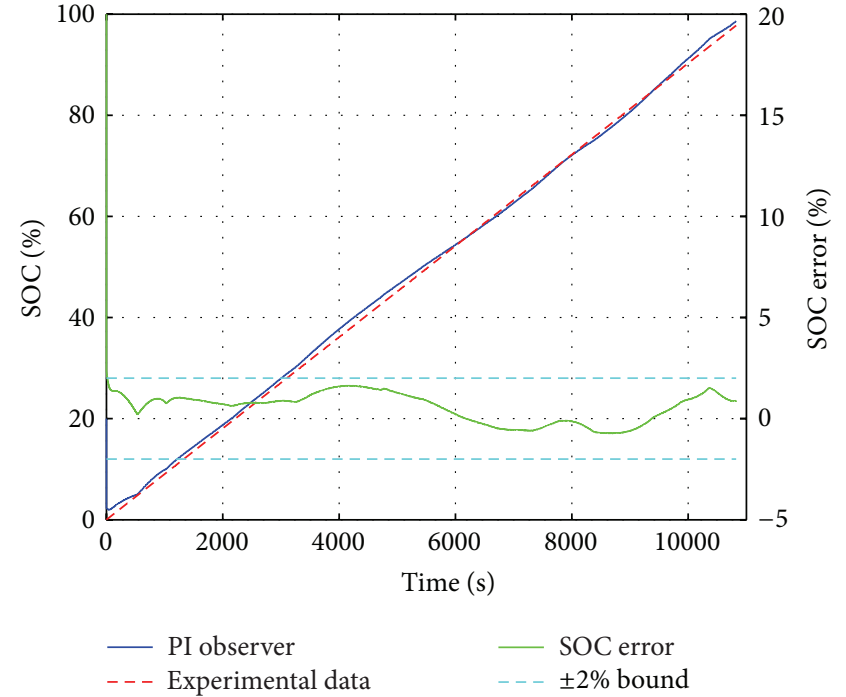

(b) PI observer

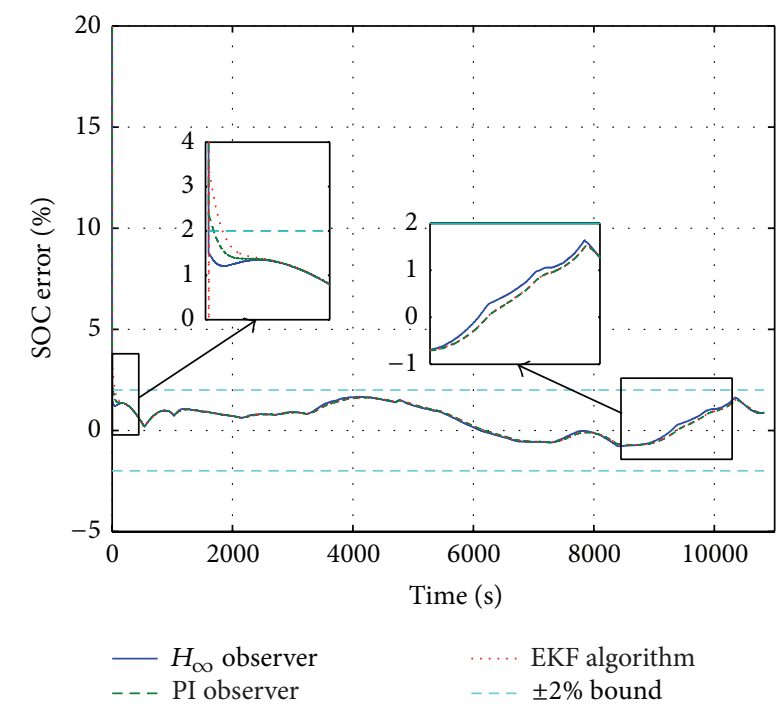

(d) Three estimation methods

FIgURE 6: The SOC estimation results when initial SOC error is $20 \%$.

adaptability of the model noise is discussed in Section 4.1, and the adaptability of the measurement noise is analyzed in Section 4.2.

4.1. The Model Parameter Perturbation. When the capacity of the battery with nominal capacity $92 \mathrm{Ah}$ declines to $74.5 \mathrm{Ah}$, other parameters of the battery will change too. If the parameters for the new battery of capacity $92 \mathrm{Ah}$ are used to estimate the SOC of the old battery of capacity $74.5 \mathrm{Ah}$, the SOC estimation accuracy of the three estimation methods will be affected significantly. Therefore, the impacts of the inaccurate battery parameters caused by battery aging to the SOC estimation accuracy are of essential importance.

In order to analyze the effects of parameter variations on SOC estimation accuracy, four cases are considered in our simulation.
Case 1. It is the process of estimating the SOC of the old battery using the parameters of the new battery.

Figure 7 shows the results of the SOC estimation in Case 1 using three algorithms. Using the $H_{\infty}$ observer, the maximum value of the SOC estimation error is $6.39 \%$, and the minimum value of the SOC estimation error is $0.4 \%$. Using the PI observer, the maximum value of the SOC estimation error is $6.33 \%$, and the minimum value of the SOC estimation error is $0.42 \%$. Using the EKF algorithm, the maximum value of the SOC estimation error is $6.33 \%$, and the minimum value of the SOC estimation error is $1.54 \%$.

Case 2. It is the process of estimating the SOC of the old battery by updating the battery's parameters used in Case 1 to the old battery's parameters except the SOC-OCV curve. 


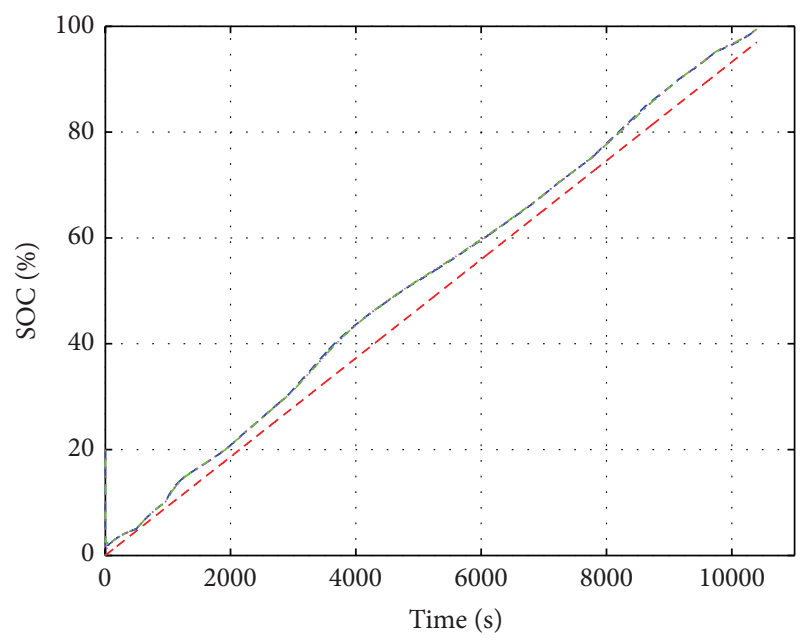

- - - Experimental data

- - $H_{\infty}$ observer

(a) SOC curves with three algorithms and experiment

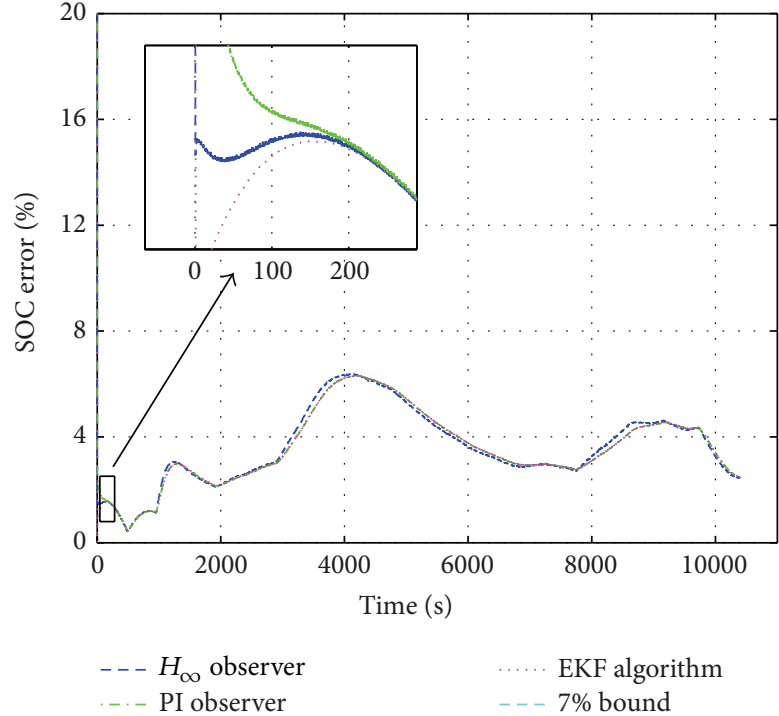

(b) SOC estimation error curves

FIGURE 7: All parameters are the new battery's ones.

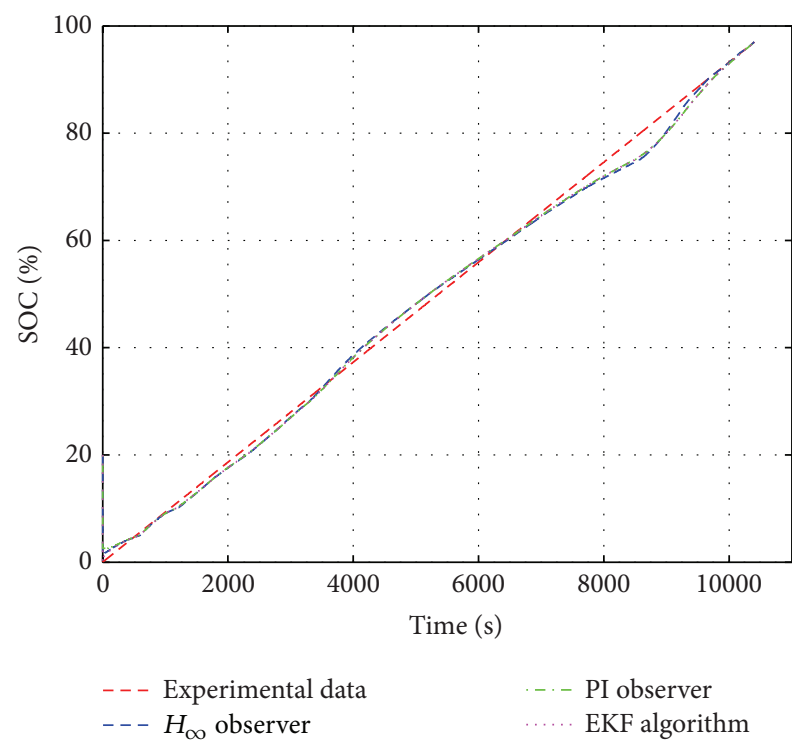

(a) SOC curves with three algorithms and experiment

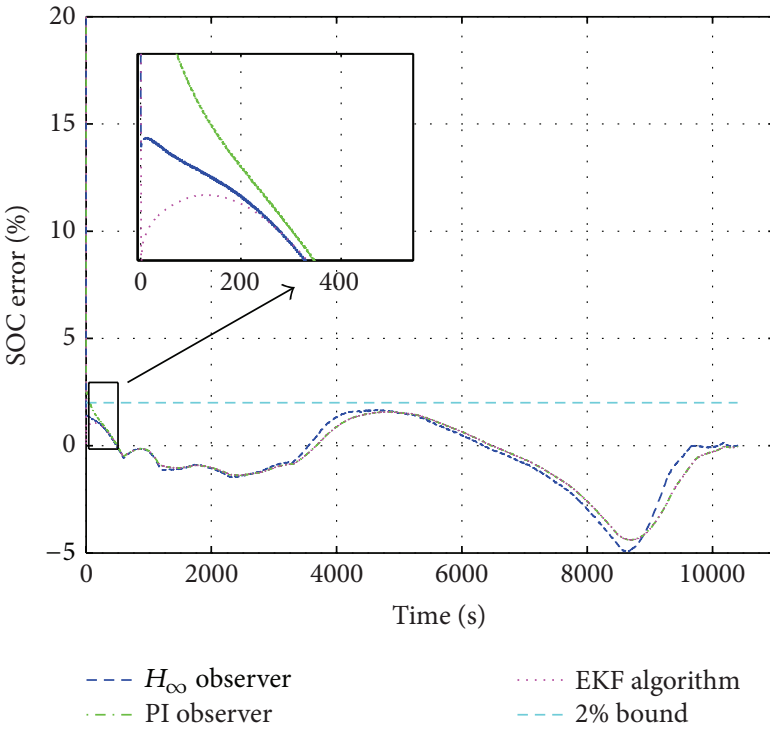

(b) SOC estimation error curves

FIgURE 8: Only the OCV is the new battery's one.

Figure 8 shows the results of the SOC estimation in Case 2 using three algorithms. Using the $H_{\infty}$ observer, the maximum value of the SOC estimation error is $1.69 \%$, and the minimum value of the SOC estimation error is $-4.96 \%$. Using the PI observer, the maximum value of the SOC estimation error is $1.59 \%$, and the minimum value of the SOC estimation error is $-4.42 \%$. Using the EKF algorithm, the maximum value of the SOC estimation error is $1.59 \%$, and the minimum value of the SOC estimation error is $-4.41 \%$.

Case 3. It is the process of estimating the SOC of the old battery by updating only the SOC-OCV curve to the old battery's parameters, while the other parameters are the same as in Case 1.

Figure 9 shows the results of the SOC estimation in Case 3 using three algorithms. Using the $H_{\infty}$ observer, the maximum value of the SOC estimation error is $8.54 \%$, and the minimum value of the SOC estimation error is $0.38 \%$. Using the PI observer, the maximum value of the SOC estimation error is $8.38 \%$, and the minimum value of the SOC estimation error is $0.40 \%$. Using the EKF algorithm, the maximum value of the SOC estimation error is $8.38 \%$, and the minimum value of the SOC estimation error is $1.40 \%$. 


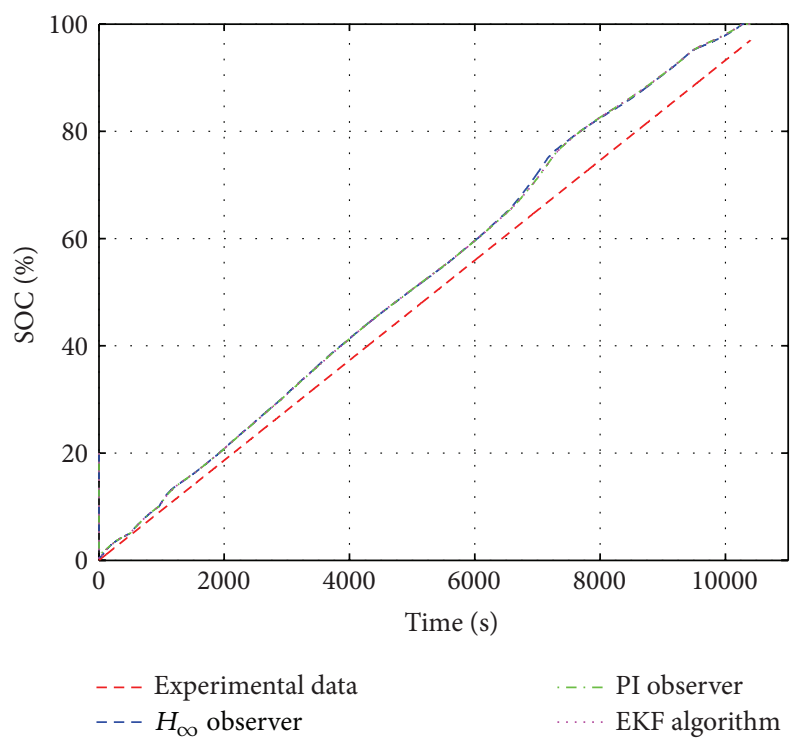

(a) SOC curves with three algorithms and experiment

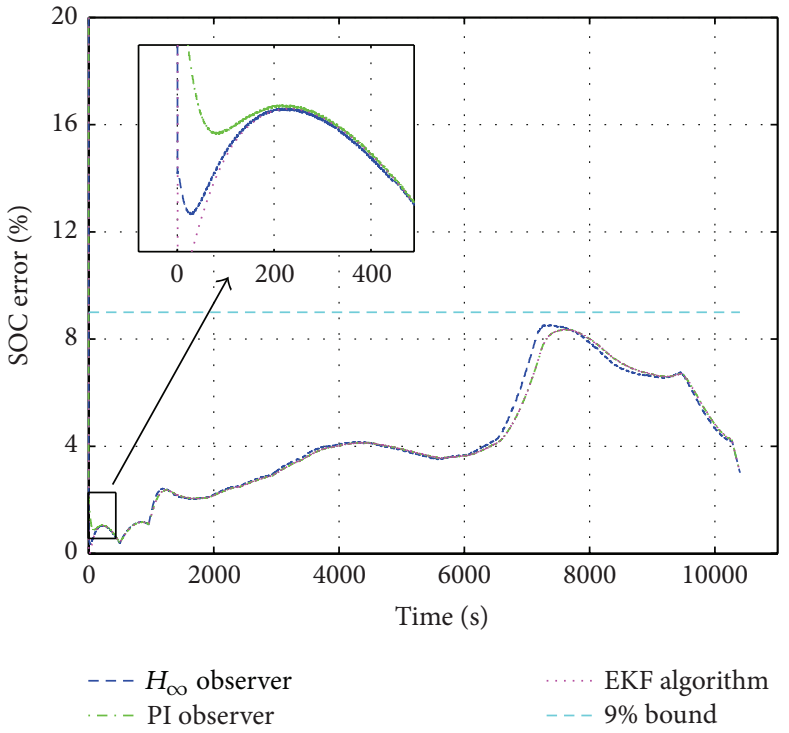

(b) SOC estimation error curves

FIGURE 9: Only the OCV is the old battery's one.

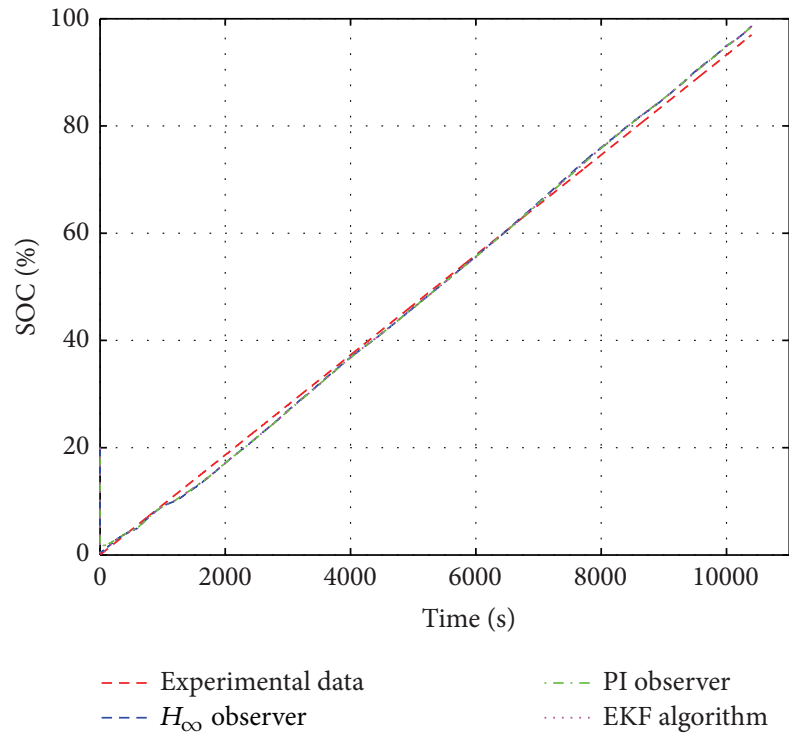

(a) SOC curves with three algorithms and experiment

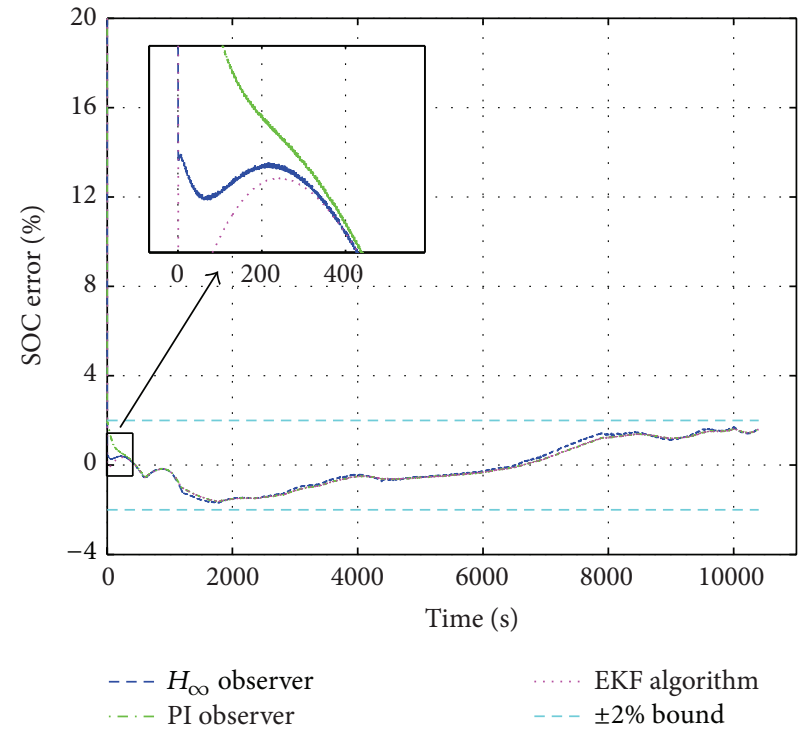

(b) SOC estimation error curves

Figure 10: All parameters are the old battery's ones.

Case 4. It is the process of estimating the SOC of the old battery by updating all the parameters to the old battery's parameters.

Figure 10 shows the results of the SOC estimation in Case 4 using three algorithms. Using the $H_{\infty}$ observer, the maximum value of the SOC estimation error is $1.72 \%$, and the minimum value of the SOC estimation error is $-1.69 \%$. Using the PI observer, the maximum value of the SOC estimation error is $1.64 \%$, and the minimum value of the SOC estimation error is $-1.63 \%$. Using the EKF algorithm, the maximum value of the SOC estimation error is $1.63 \%$, and the minimum value of the SOC estimation error is $-1.62 \%$.

Discussions. Note that

$$
\begin{aligned}
y_{\mathrm{est}} & =U_{\mathrm{OCV}}(\mathrm{SOC})+V_{P}+I R_{\mathrm{O}}, \\
\Delta y & =y_{\mathrm{exp}}-y_{\mathrm{est}}, \\
\Delta \mathrm{SOC} & =\int_{0}^{t}\left(\frac{1}{Q} I+L_{2} \Delta y\right) d t,
\end{aligned}
$$


TABLE 3: The parameters changes.

\begin{tabular}{lccc}
\hline & The maximum & The minimum & The average \\
\hline$\Delta R_{\mathrm{O}}=R_{\mathrm{O}_{-} \text {old }}-R_{\mathrm{O}_{\text {new }}}=f(\mathrm{SOC})$ & $0.87 \mathrm{~m} \Omega$, & $0.20 \mathrm{~m} \Omega$, & $0.28 \mathrm{~m} \Omega$, \\
$\Delta R_{P}=R_{P_{\text {_old }}}-R_{P_{\text {new }}}=f(\mathrm{SOC})$ & $1.6 \mathrm{~m} \Omega$, & $0.31 \mathrm{~m} \Omega$, & $0.64 \mathrm{~m} \Omega$, \\
$\Delta C_{P}=C_{P_{\text {_old }}}-C_{P_{\text {new }}}=f(\mathrm{SOC})$ & $5730 \mathrm{~F}$ & $-14968 \mathrm{~F}$ & $-604 \mathrm{~F}$ \\
$\Delta \mathrm{OCV}=\mathrm{OCV}_{\text {old }}-\mathrm{OCV}_{\text {new }}=f(\mathrm{SOC})$ & $106 \mathrm{mV}$ & $-14.3 \mathrm{mV}$ & $2.5 \mathrm{mV}$ \\
\hline
\end{tabular}

where $y_{\exp }$ is the measured terminal voltage and $y_{\text {est }}$ is the estimated terminal voltage.

From (21), since the measured terminal voltage $y_{\exp }$ is known, the estimated terminal voltage $y_{\text {est }}$ determining the terminal voltage error $\Delta y$ affects the accuracy and convergence time of the three SOC estimation methods. The estimated terminal voltage $y_{\text {est }}$ includes open circuit voltage $U_{\text {OCV }}(\mathrm{SOC})$, polarization voltage $V_{P}$, and $I R_{O}$, as (20) shows. Therefore, they have obvious effects on estimation accuracy and robustness of the three SOC estimation methods, as (22) shows, demonstrated by Figures 7-10.

The parameters changes from the new battery to the old battery's parameters are shown as in Table 3.

When the battery is aging from $92 \mathrm{Ah}$ to $74.5 \mathrm{Ah}$, during a complete charging process in $1 / 3 \mathrm{C}$ rate, the average polarization voltage increase is $16 \mathrm{mV}$, and the average Ohmic voltage increase is $7 \mathrm{mV}$. However, the average OCV decrease is $2.5 \mathrm{mV}$.

When we use the parameters of the old battery to estimate the SOC of the old battery, the SOC estimation error is only caused by the battery model error. The terminal voltage error caused by the battery model is considered to be $\Delta$, and the SOC estimation result is shown in Figure 10.

However, using the parameters of the new battery to estimate the SOC of the old battery, which means that the parameters are not updated, the estimated value of the terminal voltage $y_{\text {est }}$ is $(\Delta+20.5) \mathrm{mV}$ smaller than the measured terminal voltage value. This causes a great SOC estimation error, whose maximum $E 1 \_$max is between $2 \%$ and $8 \%$, as shown in Figures 7 and 10 . By updating the Ohmic resistance and the polarization resistance to the true values of the old battery, the estimated value of the terminal voltage $y_{\text {est }}$ is $(\Delta-2.5) \mathrm{mV}$ smaller than the measured terminal voltage value. This causes only a little SOC estimation error, whose minimum $E 2 \_$min is less than $-2 \%$, as shown in Figures 8 and 10. If the OCV is updated, but not other parameters, to the value of the old battery, the estimated value of the terminal voltage $y_{\text {est }}$ is $(\Delta+23) \mathrm{mV}$ smaller than the measured terminal voltage value. Because $(\Delta+23) \mathrm{mV}$ is larger than $(\Delta+20.5) \mathrm{mV}$, estimation errors by using the updated OCV of old battery are worst among all cases, whose maximum E3_max is more than 8\%, larger than E1_max, as shown in Figures 9 and 10. The clear comparison is shown in Table 4.

In our recent studies [33], it is shown that SOC estimation accuracy is dependent on the curve of SOC-OCV significantly, if the SOC-OCV curve varies substantially. In this study during the progress of the battery aging, the SOC-OCV curve does not change much. The experimental results are included in Figure 11. As a result, the impact of aging on the

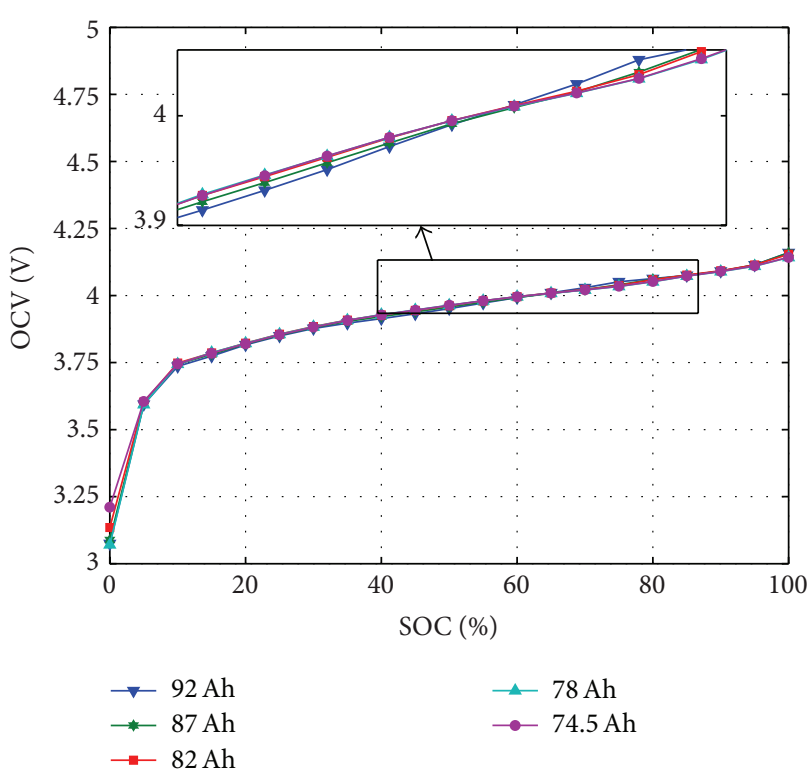

FIGURE 11: OCV-SOC curves at different aging states.

OCV has negligible effect on SOC estimation accuracy, as analyzed in Section 4.1. In contrast, effect of aging on other model parameters plays much more prominent roles in SOC estimation accuracy.

In addition, we may conclude from comparing Figures 7, 8,9 , and 10 that the SOC estimation error when using the parameters of the new battery to estimate the SOC of the old battery is not a linear superposition of the SOC estimation error caused by the changes of the resistance and capacitance and the changes of the curve of SOC-OCV. This is clearly indicated in Figure 12, taking the SOC estimation results of the PI observer as an example.

4.2. The Terminal Voltage Measurement Errors. In order to find the relationship between the SOC estimation accuracy and the statistical characteristics of the terminal voltage measurement noise, we further add measurement noises with different means and variances to the experimental data of the terminal voltage in Section 3.5. There are different SOC estimation results using different estimation methods.

(i) The $H_{\infty}$ Observer. When the mean of the measurement noise changes from $-6 \mathrm{mV}$ to $4 \mathrm{mV}$ and standard deviation of the measurement noise increases from 0 to $10 \mathrm{mV}$, the SOC accuracy is depicted in Figure 13. 
TABLE 4: Terminal voltage error and SOC estimation error.

\begin{tabular}{lcc}
\hline Cases estimating SOC of the old battery & $\Delta y=y_{\text {exp }}-y_{\text {est }}$ & SOC estimation error \\
\hline Case 1: all parameters are the new battery's ones & $(\Delta+22.5) \mathrm{mV}$ & $2 \%<E 1 \_\mathrm{max}<8 \%$ \\
Case 2: only the OCV is the new battery's one & $(\Delta-2.5) \mathrm{mV}$ & $-5 \%<E 2 \_\mathrm{min}<-2 \%$ \\
Case 3: only the OCV is the old battery's one & $(\Delta+23) \mathrm{mV}$ & $E 1 \_\mathrm{max}<8 \%<E 3 \_\mathrm{max}$ \\
Case 4: all parameters are the old battery's ones & $\Delta \mathrm{mV}$ & $-2 \%<E 4<2 \%$ \\
\hline
\end{tabular}

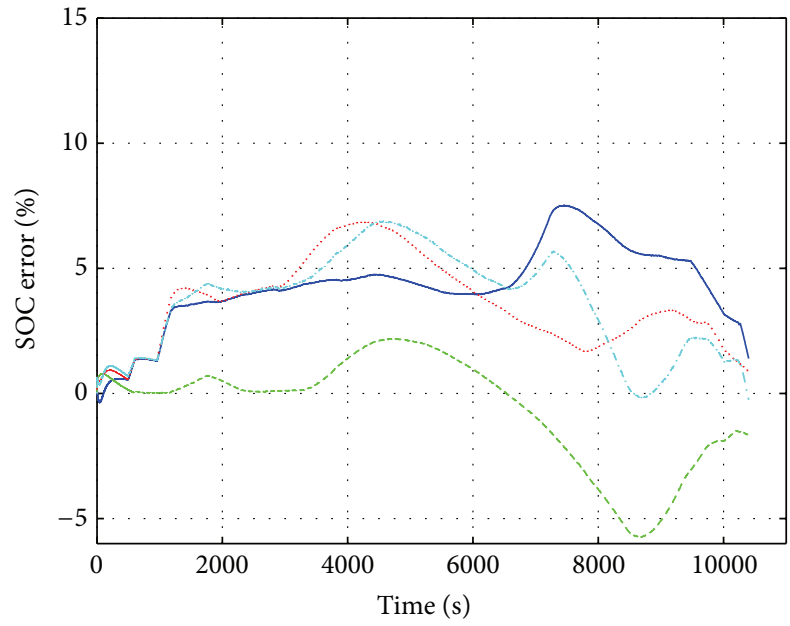

_ SOC error in case 3 - SOC error in case 4

- - - SOC error in case 2 - SOC error in case 4

..... SOC error in case $1-$ SOC error in case 4

-.- Green line + blue line

FIgURE 12: The comparison of the SOC estimation errors.

If the SOC estimation error is required to be less than $2 \%$, the mean and standard deviation of the measurement noise must be confined from $-3.172 \mathrm{mV}$ to $1.172 \mathrm{mV}$ and 0 to $3.43 \mathrm{mV}$, respectively.

(ii) The PI Observer. When the mean of the measurement noise changes from $-6 \mathrm{mV}$ to $4 \mathrm{mV}$ and standard deviation of the measurement noise increases from 0 to $10 \mathrm{mV}$, the SOC accuracy is shown in Figure 14.

If the SOC estimation error is required to be less than $2 \%$, the mean and standard deviation of the measurement noise must be confined from $-3.36 \mathrm{mV}$ to $1.172 \mathrm{mV}$ and 0 to $5.15 \mathrm{mV}$, respectively.

(iii) The EKF Algorithm. When the mean of the measurement noise changes from $-6 \mathrm{mV}$ to $4 \mathrm{mV}$ and standard deviation of the measurement noise increases from 0 to $10 \mathrm{mV}$, the SOC accuracy is illustrated in Figure 15.

If the SOC estimation error is required to be less than $2 \%$, the mean and standard deviation of the measurement noise must be confined from $-3.36 \mathrm{mV}$ to $1.3 \mathrm{mV}$, 0 to $3.535 \mathrm{mV}$, respectively.

(iv) Discussions. The principle of the $H_{\infty}$ observer is similar to the Luenberger observer, whose feedback gain is a proportional gain. It controls quite aggressively with an expected fast

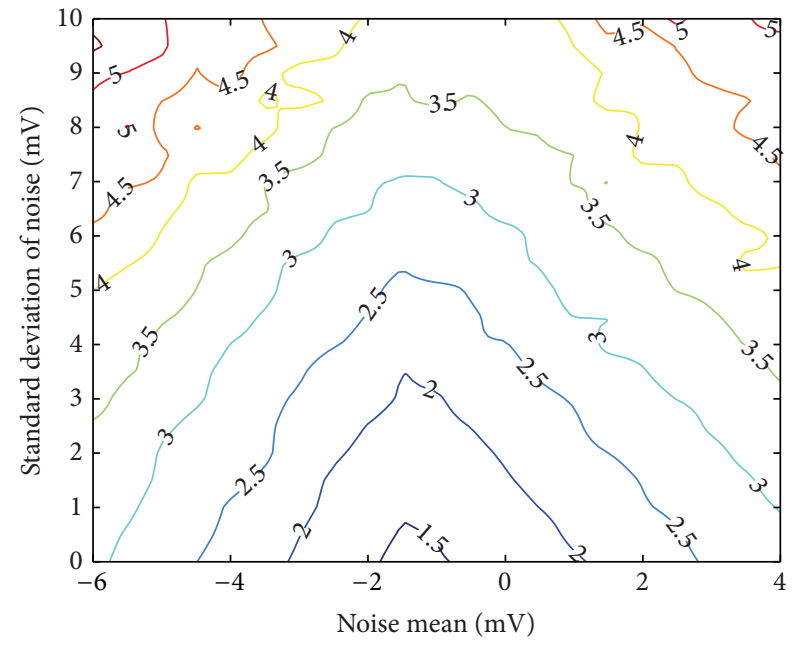

(a) SOC error

FIGURE 13: The SOC estimation error of the $H_{\infty}$ observer when statistical characteristics of the noise change.

response. However, it shows certain levels of overreaction, causing nonsmooth contours of SOC estimation errors. In contrast the PI observer with its integral part acts as a signal smoother (a low-pass filter type), so the contours of its SOC estimation errors are smoother than that of the $H_{\infty}$ observer. Note that the gain of the EKF algorithm is updated timely, so the contours of its SOC estimation errors are the smoothest.

To capture transient errors more concretely, in Figures 1315 , we define the area enclosed by the $x$-axis and the contour line as the total absolute error. The total absolute error ratio is then calculated as the total absolute error divided by the area which is equal to the product of the maximum value of $x$-axis and maximum value of $y$-axis in the figure. The ratios of the three observers are shown in Figure 16.

Under the same SOC estimation accuracy, the adaptation range against the measurement noise of the PI observer is the biggest and that of the $H_{\infty}$ observer is the smallest, as Figure 16 shows. It is worth noting that Figures 13, 14, and 15 are not symmetrical about the mean, result from the total effect of the terminal voltage noise mean and the inaccurate parameters on the SOC estimation accuracy.

\section{Conclusions}

In this paper, several typical SOC estimation algorithms including the $H_{\infty}$ observer, PI observer, and extended 


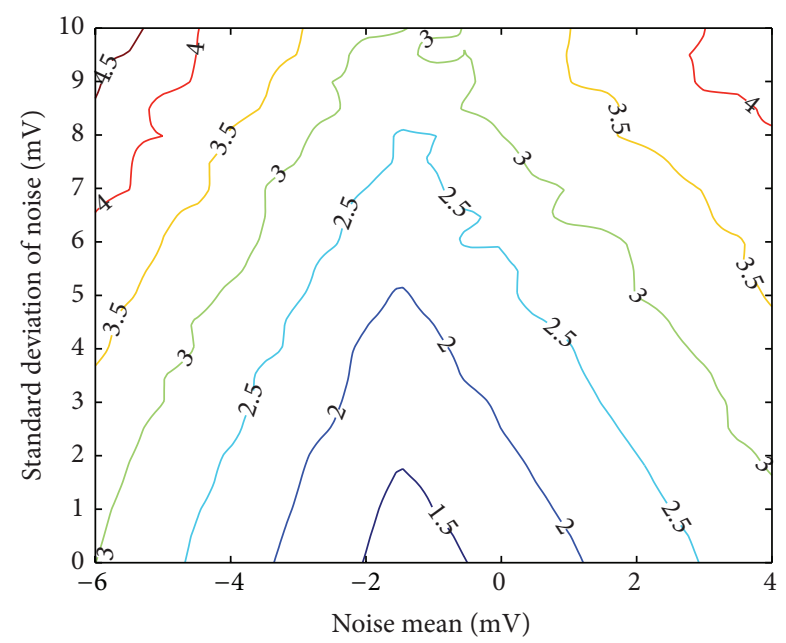

(a) SOC error

FIGURE 14: The SOC estimation error of the PI observer when statistical characteristics of the noise change.

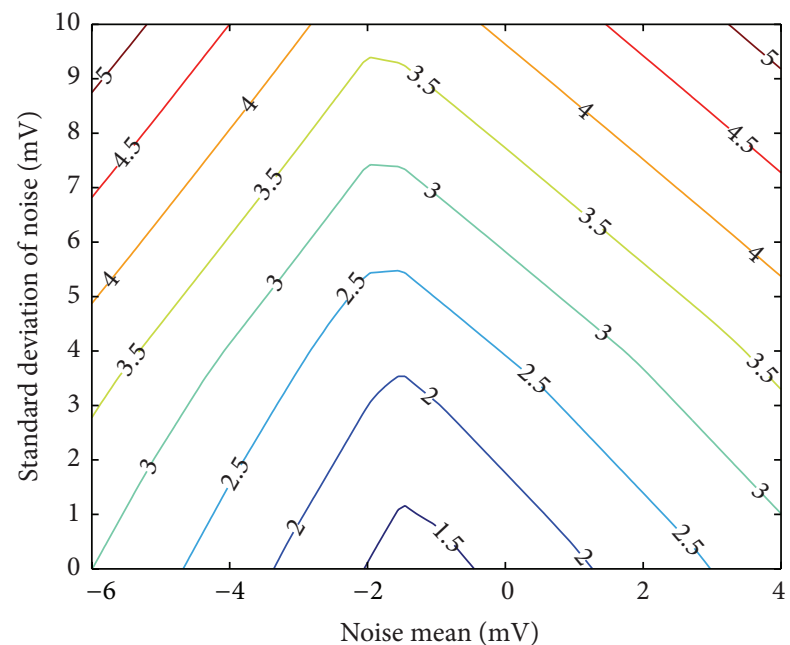

(a) SOC error

FIGURE 15: The SOC estimation error of the EKF observer when statistical characteristics of the noise change.

Kalman filter are applied to estimate SOC. By considering four categories of variations of model parameters caused by battery aging and studying the influence of each category on the SOC estimation precision, we compare the algorithms in terms of their robustness against modeling errors. In addition, their tolerance to voltage measurement errors is quantitatively evaluated.

The robustness and the estimation accuracy of the three methods against modeling errors and measurement noises are similar. However, the $H_{\infty}$ observer needs to know the gain $L$, which needs to calculate the Linear Matrix Inequalities in advance; the EKF algorithm needs to know the distribution of the measurement noise, which is difficult to be obtained

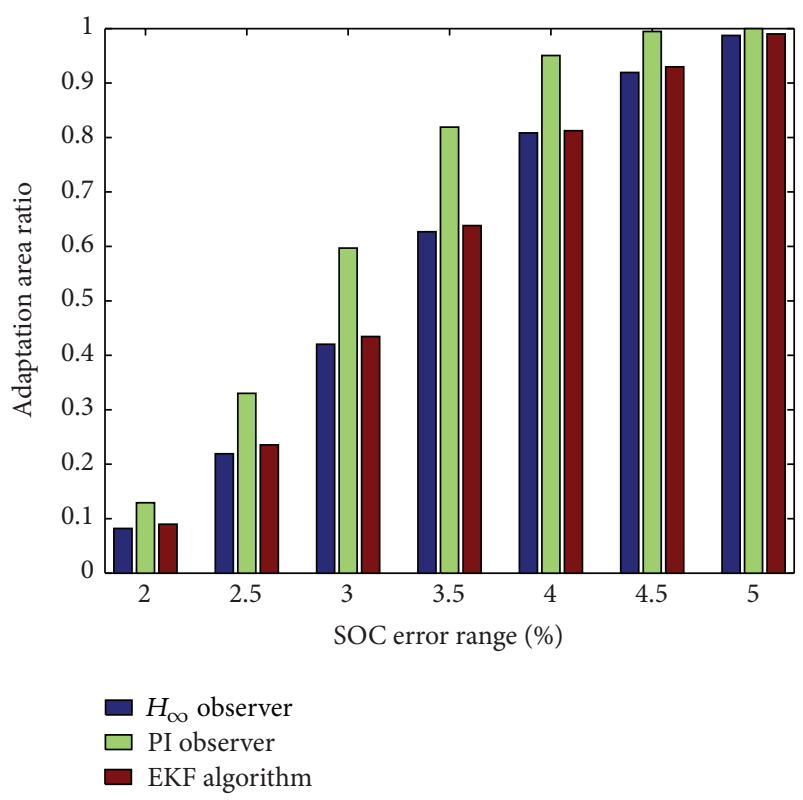

FIGURE 16: The total absolute error ratio of the measurement noise.

in fact. Compared to these two methods, the PI observer can acquire the proportional gain $K_{p}$ and the integral gain $K_{i}$ depending on the experiences. Therefore, in the view of application and SOC accuracy, the PI observer has advantages over the $H_{\infty}$ observer and the EKF algorithm to be applied in BMS. Through simulation results we reach the following conclusions:

(1) The Ohmic resistance $R_{O}$, polarization resistance $R_{P}$, and the open circuit voltage OCV are the key parameters affecting SOC estimation accuracy. However, the polarization capacitor $C_{P}$, which is an important parameter, only influences the dynamic response characteristics of SOC estimation but does not have noticeable effects on the steady-state accuracy of SOC estimation.

(2) Under the same SOC estimation accuracy and the robustness against modeling errors and measurement noises, the PI observer has advantages over the $H_{\infty}$ observer and the EKF algorithm to be applied in BMS.

(3) The relationship between SOC estimation accuracy and voltage measurement errors has been resolved, and some related guidelines on how to select a robust method which has a strong tolerance against voltage measurement errors are provided.

There are several important related topics that are not covered in this paper. First, optimal design of PI observers requires essential statistical information on measurement noises and individualized models. Learning algorithms for noise characterizations and parameter estimation can lead to adaptive PI observers with improved SOC estimation accuracy. Furthermore, implementation of SOC estimators in battery management systems on electric vehicles encounters hardware and computational complexity constraints. For 
example, sensor precision levels and sampling rates will limit data flow rates and reliability. Analysis of the influence of synchronous sampling, sampling rates, asynchronous sampling, and quantization on SOC estimation accuracy will be pursued in our future studies.

\section{Conflict of Interests}

The authors declare that there is no conflict of interests regarding the publication of this paper.

\section{Acknowledgment}

The work was supported by National Natural Science Foundation of China under Grant no. 51477009.

\section{References}

[1] K. W. E. Cheng, B. P. Divakar, H. Wu, K. Ding, and H. F. Ho, "Battery-management system (BMS) and SOC development for electrical vehicles," IEEE Transactions on Vehicular Technology, vol. 60, no. 1, pp. 76-88, 2011.

[2] V. Pop, H. J. Bergveld, D. Danilov, P. P. L. Regtien, and P. H. L. Notten, Battery Management Systems: Accurate State-ofCharge Indication for Battery-Powered Applications, Springer, New York, NY, USA, 2008.

[3] S. Piller, M. Perrin, and A. Jossen, "Methods for state-of-charge determination and their applications," Journal of Power Sources, vol. 96, no. 1, pp. 113-120, 2001.

[4] S. Lee, J. Kim, J. Lee, and B. H. Cho, "State-of-charge and capacity estimation of lithium-ion battery using a new opencircuit voltage versus state-of-charge," Journal of Power Sources, vol. 185, no. 2, pp. 1367-1373, 2008.

[5] J. Xu, C. C. Mi, B. Cao, and J. Cao, "A new method to estimate the state of charge of lithium-ion batteries based on the battery impedance model," Journal of Power Sources, vol. 233, pp. 277284, 2013.

[6] M. Mastali, J. Vazquez-Arenas, R. Fraser, M. Fowler, S. Afshar, and M. Stevens, "Battery state of the charge estimation using Kalman filtering," Journal of Power Sources, vol. 239, pp. 294307, 2013.

[7] C. P. Zhang, J. C. Jiang, W. G. Zhang, and S. M. Sharkh, "Estimation of state of charge of lithium-ion batteries used in HEV using robust extended Kalman filtering," Energies, vol. 5, no. 4, pp. 1098-1115, 2012.

[8] Z. Chen, Y. Fu, and C. C. Mi, "State of charge estimation of lithium-ion batteries in electric drive vehicles using extended Kalman filtering," IEEE Transactions on Vehicular Technology, vol. 62, no. 3, pp. 1020-1030, 2013.

[9] R. Xiong, H. He, F. Sun, and K. Zhao, "Evaluation on state of charge estimation of batteries with adaptive extended kalman filter by experiment approach," IEEE Transactions on Vehicular Technology, vol. 62, no. 1, pp. 108-117, 2013.

[10] G. L. Plett, "Extended Kalman filtering for battery management systems of LiPB-based HEV battery packs: Part 3. State and parameter estimation," Journal of Power Sources, vol. 134, no. 2, pp. 277-292, 2004.

[11] G. L. Plett, "Extended Kalman filtering for battery management systems of LiPB-based HEV battery packs: part 2. Modeling and identification," Journal of Power Sources, vol. 134, no. 2, pp. 262276, 2004.
[12] G. L. Plett, "Extended Kalman filtering for battery management systems of LiPB-based HEV battery packs. Part 1. Background," Journal of Power Sources, vol. 134, no. 2, pp. 252-261, 2004.

[13] F. Zhang, G. Liu, L. Fang, and H. Wang, "Estimation of battery state of charge with $H_{\infty}$ observer: applied to a robot for inspecting power transmission lines," IEEE Transactions on Industrial Electronics, vol. 59, no. 2, pp. 1086-1095, 2012.

[14] X. Hu, S. Li, H. Peng, and F. Sun, "Robustness analysis of Stateof-Charge estimation methods for two types of Li-ion batteries," Journal of Power Sources, vol. 217, pp. 209-219, 2012.

[15] M. Chen and G. A. Rincón-Mora, "Accurate electrical battery model capable of predicting runtime and I-V performance," IEEE Transactions on Energy Conversion, vol. 21, no. 2, pp. 504511, 2006.

[16] L. Gao, S. Liu, and R. A. Dougal, "Dynamic lithium-ion battery model for system simulation," IEEE Transactions on Components and Packaging Technologies, vol. 25, no. 3, pp. 495$505,2002$.

[17] V. H. Johnson, "Battery performance models in ADVISOR," Journal of Power Sources, vol. 110, no. 2, pp. 321-329, 2002.

[18] T.-S. Dao, C. P. Vyasarayani, and J. McPhee, "Simplification and order reduction of lithium-ion battery model based on porouselectrode theory," Journal of Power Sources, vol. 198, pp. 329-337, 2012.

[19] A. Szumanowski and Y. Chang, "Battery management system based on battery nonlinear dynamics modeling," IEEE Transactions on Vehicular Technology, vol. 57, no. 3, pp. 1425-1432, 2008.

[20] H. He, R. Xiong, X. Zhang, F. Sun, and J. Fan, "State-ofcharge estimation of the lithium-ion battery using an adaptive extended kalman filter based on an improved Thevenin model," IEEE Transactions on Vehicular Technology, vol. 60, no. 4, pp. 1461-1469, 2011.

[21] G. L. Wu, R. G. Lu, C. Zhu, and C. C. Chan, "An improved Ampere-hour method for battery state of charge estimation based on temperature, coulomb efficiency model and capacity loss model," in Proceedings of the IEEE Vehicle Power and Propulsion Conference (VPPC '10), pp. 1-4, Lille, France, September 2010.

[22] M.-H. Chang, H.-P. Huang, and S.-W. Chang, "A new state of charge estimation method for $\mathrm{LiFePO}_{4}$ battery packs used in robots," Energies, vol. 6, no. 4, pp. 2007-2030, 2013.

[23] G. Zames, "Feedback and optimal sensitivity: model reference transformations, multiplicative seminorms, and approximate inverses," IEEE Transactions on Automatic Control, vol. 26, no. 2, pp. 301-320, 1981

[24] J. Huang and C.-F. Lin, "Numerical approach to computing nonlinear $H_{\infty}$ control laws," Journal of Guidance, Control, and Dynamics, vol. 18, no. 5, pp. 989-996, 1995.

[25] J. C. Doyle, K. Glover, P. P. Khargonekar, and B. A. Francis, "State-space solutions to standard $\mathrm{H}_{2}$ and $H_{\infty}$ control problems," IEEE Transactions on Automatic Control, vol. 34, no. 8, pp. 831-847, 1989.

[26] J. W. Helton and A. Sideris, "Frequency response algorithms for $H_{\infty}$ optimization with time domain constraints," IEEE Transactions on Automatic Control, vol. 34, no. 4, pp. 427-434, 1989.

[27] K. Zhou and J. Doyle, Essentials of Robust Control, Prentice Hall, Hoboken, NJ, USA, 1998.

[28] J. Xu, C. C. Mi, B. Cao, J. Deng, Z. Chen, and S. Li, “The state of charge estimation of lithium-ion batteries based on a proportional-integral observer," IEEE Transactions on Vehicular Technology, vol. 63, no. 4, pp. 1614-1621, 2014. 
[29] N. B. Nichols and J. G. Ziegler, "Optimum settings for automatic controllers," Journal of Dynamic Systems, Measurement, and Control, vol. 115, no. 2, pp. 220-222, 1993.

[30] R. E. Kalman, "A new approach to linear filtering and prediction problems," Journal of Fluids Engineering, vol. 82, no. 1, pp. 35-45, 1960.

[31] B. Ristic, S. Arulampalam, and N. Gordon, Beyond the Kalman Filter, Artech House, Boston, Mass, USA, 2004.

[32] M. Hoshiya and E. Saito, "Structural identification by extended Kalman filter," Journal of Engineering Mechanics, vol. 110, no. 12, pp. 1757-1770, 1984.

[33] C. P. Zhang, L. Y. Wang, X. Li et al., "Robust and adaptive estimation of state of charge for lithium-ion batteries," IEEE Transactions on Industrial Electronics, vol. 62, no. 8, pp. 49484957, 2015. 


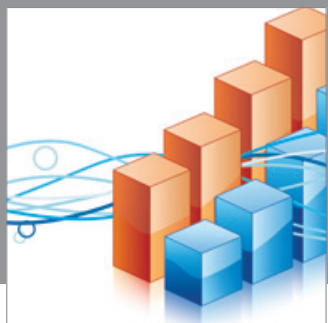

Advances in

Operations Research

mansans

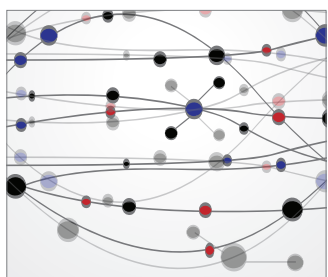

The Scientific World Journal
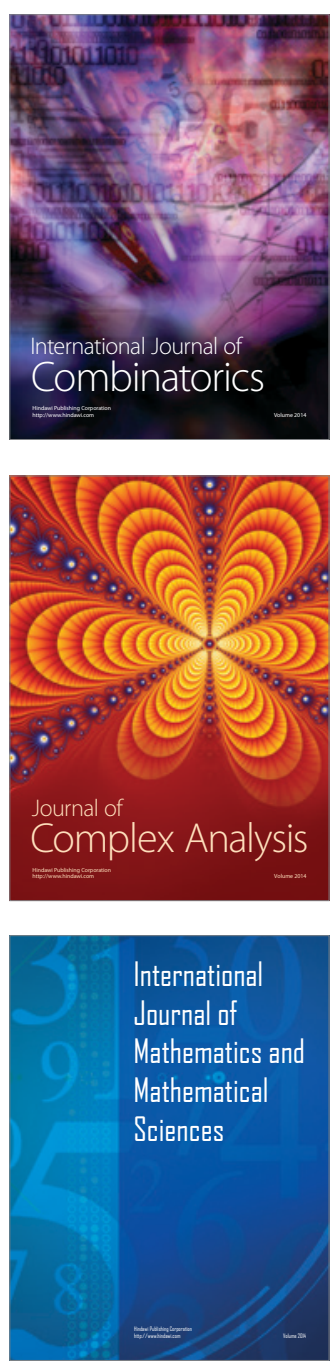
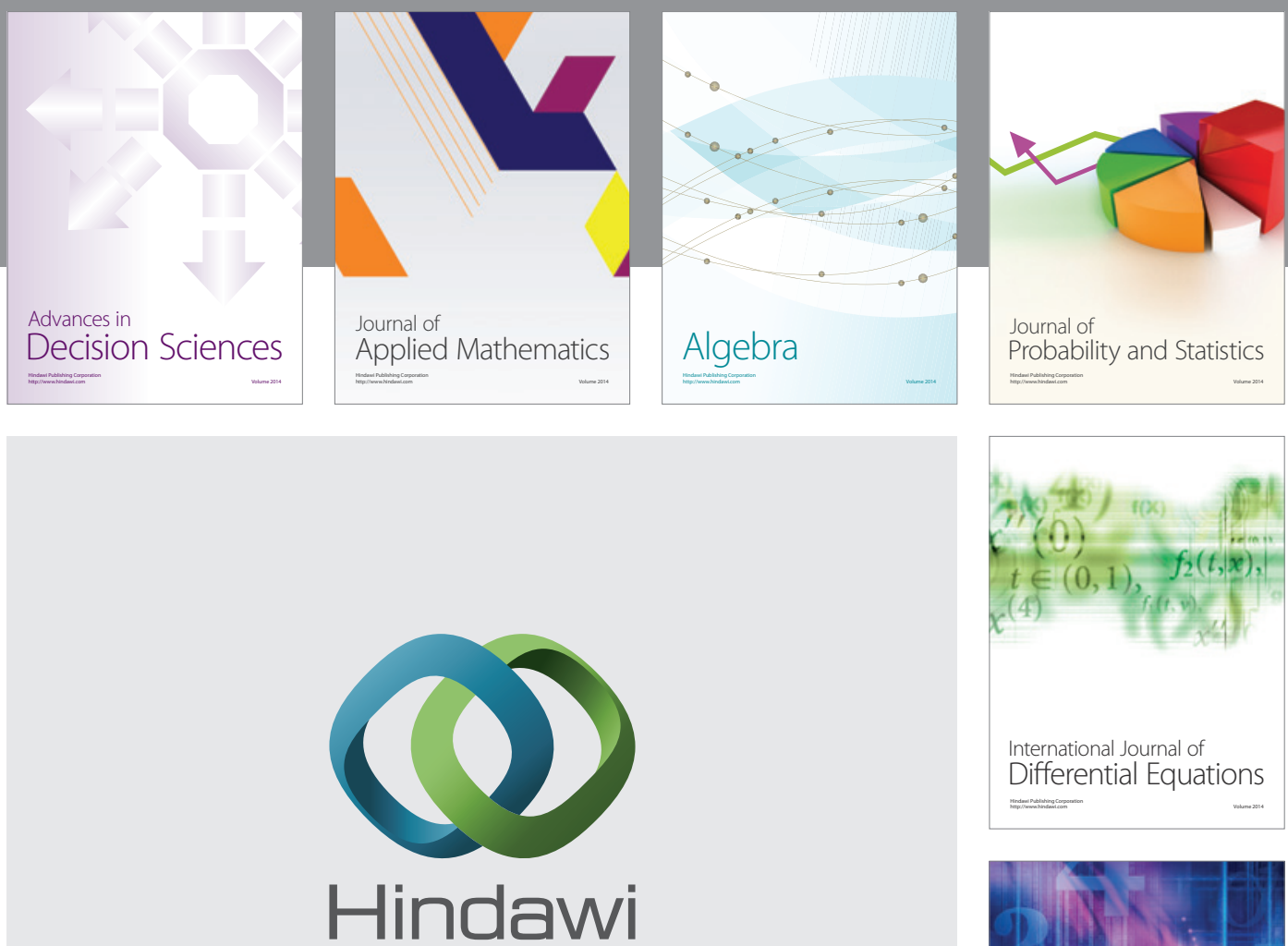

Submit your manuscripts at http://www.hindawi.com
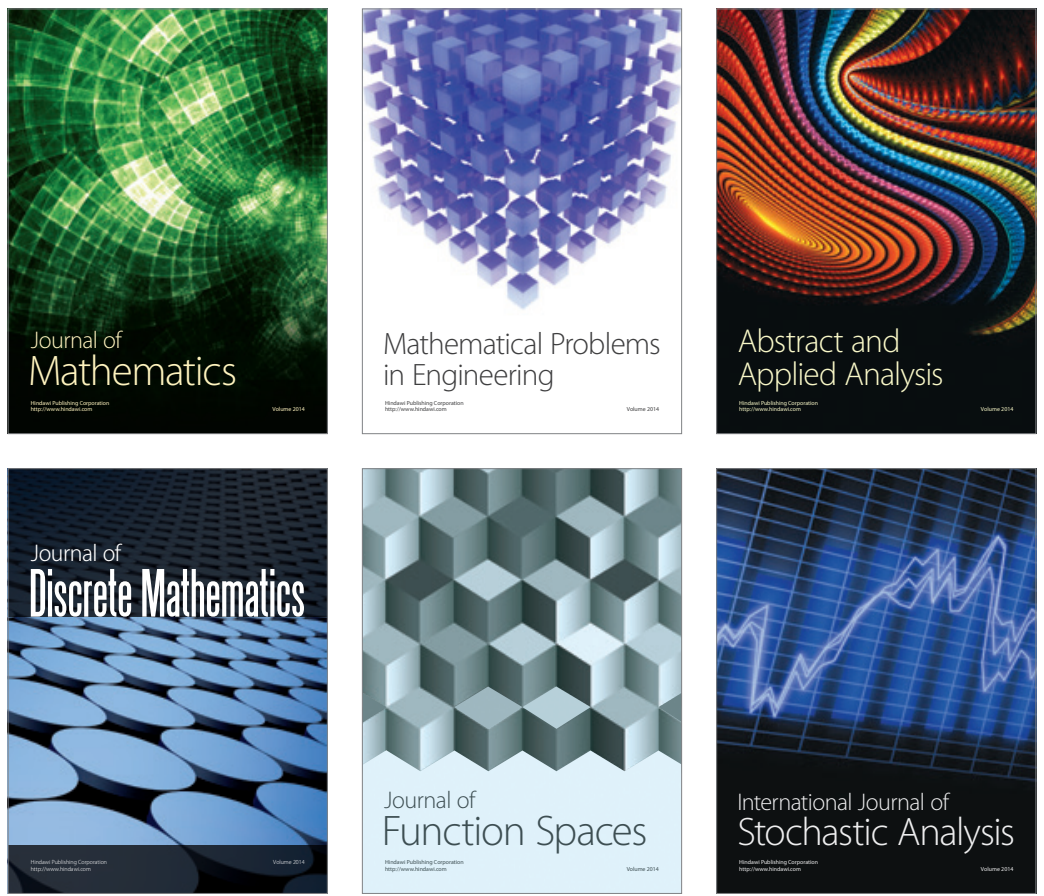

Journal of

Function Spaces

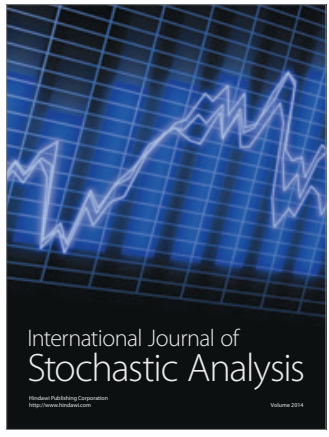

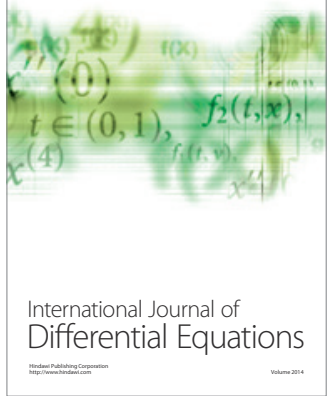
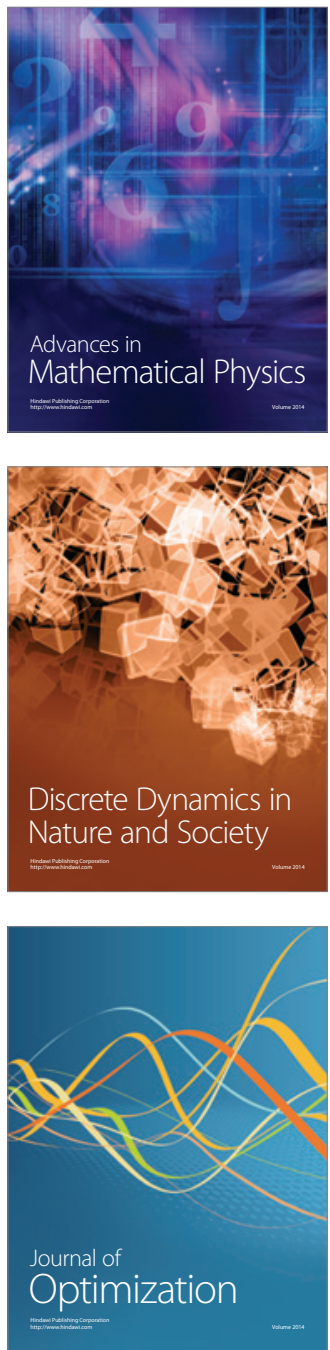